\title{
Rarefied Gas Flows in Micro-Channels
}

\author{
Chong Xie, Jing Fan and Ching Shen
}

Laboratory for High-Temperature Gas Dynamics, Institute of Mechanics, CAS, Beijing 100080, China

\begin{abstract}
Rarefied gas flows through micro-channels are simulated using particle approaches, named as the information preservation (IP) method and the direct simulation Monte Carlo (DSMC) method. Some important issues such as mass-flux convergence in IP calculations and the tangential momentum accommodation coefficient along micro-channel surfaces in experiment are addressed. Stream-wise pressure distributions and mass fluxes through micro-channels given by the IP method agree well with experimental data measured by Pong et al, Shih et al, and Arkilic et al, respectively.
\end{abstract}

\section{INTRODUCTION}

The gas flows in micro-channel, a basic element of MEMS devices, have been studied experimentally and theoretically. In the experiments [1-7], the typical channel dimensions were about one micron high by several tens of microns wide and by several thousands microns long. The flow was driven by the pressure difference between the inlet and outlet that led to a typical inlet velocity of about $0.2 \mathrm{~m} / \mathrm{s}$ [8]. It was observed that the stream-wise pressure profiles were non-linear. To take into account this non-continuum effect, a velocity-slip boundary condition was introduced in analytically or numerically solving the Navier-Stokes equation [2,9]. Properly choosing the tangential momentum accommodation coefficient $\sigma$, the slip Navier-Stokes solution agreed with the measured data when the Knudsen number, $K n=\lambda / H$ is smaller than 0.1 , where $\lambda$ is the molecular mean free path, and $\mathrm{H}$ is the channel height $[6,7,9]$. Kinetic theory indicates that all continuum models break down at a sufficiently high $K n$. This was also verified by further micro-channel experiment: the slope of the Navier-Stokes flow conductance obviously deviated from the experimental data at an outlet Knudsen number of about 1.95 [7].

The purpose of the present work is to provide a kinetic description of micro-channel gas flows at different experimental conditions [1,5-7]. Because of a small characteristic velocity of $0.2 \mathrm{~m} / \mathrm{s}$, conventional kinetic schemes such as the direct simulation Monte Carlo (DSMC) method faces a serious statistical noise arising from the thermal velocity. It requires a sample size of $10^{8}$ to isolate the flow velocity of $0.2 \mathrm{~m} / \mathrm{s}$ from the noise. Because the DSMC computational amount is proportional to the sample size, such an enormous size is extremely time-consuming and beyond the current computer capabilities [10]. The authors proposed a particle-based method, called the information preservation (IP) method [11,12], which was proven highly effective to reduce the statistical noise, for instance, a sample size of $10^{4}$ is enough for IP to resolve the velocity of $0.2 \mathrm{~m} / \mathrm{s}$, four orders less than that required by DSMC. The IP method has been successfully applied to benchmark problems, namely Couette flow, Poiseuille flow and 
Rayleigh flow, over the entire Knudsen regime [11,12]. The macroscopic velocity, surface shear stresses, and mass fluxes given by IP were in excellent agreement with exact solutions in the continuum and free molecular regimes, and with experimental data [13] and the linearized Boltzmann solutions [14,15] in the transition regime. Recently, Sun, Boyd and Candler [16] simulated low subsonic airflows past a flat plate of 20 microns long over the entire Knudsen regime using IP. The drag coefficient given by IP compared well with experimental data of Schaff \& Sherman [17], and Janour [18].

Application of the IP method to micro-channel flows is straightforward and has been demonstrated in preliminary studies [19,20]. For a micro-channel of $1.2 \mu \mathrm{m}$ high by $3000 \mu \mathrm{m}$ long, with the inlet and outlet pressures of 20 and 0 psig, respectively, the stream-wise pressure distribution obtained by IP agreed well with the experimental data of Pong et al [19]. Specific complications naturally arose. One of them was the accumulation of numerical error due to a large ratio of the length to height, which considerably delayed the mass-flux convergence in the calculation. In this article, we will simulate micro-channel gas flows, and compare the results with measured data available. Firstly, certain issues in IP calculation are addressed. Then the tangential momentum accommodation coefficient along micro-channel surfaces in experiment is discussed. Next, the IP method is used to simulate micro-channel gas flows at various experimental conditions. Finally, some conclusions are given.

\section{COMPUTATIONAL ISSUES}

In the IP method, a relatively small number of model molecules is stored in a computer to represent the large number of molecules in real gas flows, and each simulated molecule is assigned two velocities: thermal velocity $\vec{c}_{i}$ and information velocity $\vec{u}_{i}[11,12]$. A simulated molecule moves through physical space and undergoes collisions appropriate to the thermal velocity, following the same algorithms and models as the DSMC method [21], while the information velocity corresponds to the collective velocity of the enormous number of real molecules represented by the simulated molecule. Implementation of the IP method for multi-dimensional flows has been described in detail $[20,22,23]$, and may be briefly summarized as follows.

1) Initially, $\vec{u}_{i}$ is set based on an initial velocity field.

2) For simulated molecules diffusely reflected from a wall, $\vec{u}_{i}$ has the same velocity as the wall.

3) For simulated molecules entering the computational domain from boundaries, $\vec{u}_{i}$ is set to satisfy the boundary condition.

4) For two simulated molecules colliding each other, the post-collision velocities satisfy the momentum conservation

$$
u_{i, 1}^{*}=u_{i, 2}^{*}=\frac{m_{1} u_{i, 1}+m_{2} u_{i, 2}}{m_{1}+m_{2}},
$$

where superscript * denotes post-collision.

5) If there are external forces acting on a cell, acceleration $\vec{a}=\stackrel{\mathrm{r}}{F} / \rho \Delta V$ will contribute an velocity increment $\vec{a} \Delta t$ to each simulated molecule during a time step $\Delta t$, where $\underset{F}{F}$ is the sum of the external forces, $\rho$ and $\Delta V$ are the density and volume of the cell, respectively.

6) When there is a pressure gradient across a cell, the change of information velocity obeys the momentum equation

$$
\Delta u_{i}=-\frac{\Delta t}{\rho \Delta V} \iint p 1_{i} d S
$$


where $\lambda_{i}$ is the normal vector of the surface. The right hand side of (2) is written in a non-viscous form, rather than a more general form $-(\Delta / \rho \Delta V) \iint \sigma_{i j} l d S$, where $\sigma_{i j}$ is the stress tensor. Because the IP variables are carried by the molecules in a DSMC procedure, the transport process of viscosity and diffusion have done directly through molecular motion across the cell surfaces. Therefore, the IP method, though apparently controlled by a non-viscous momentum equation (2), essentially obeys a more universal transport relation applicable over the entire flow regime.

7) Update the velocity, density and temperature of a cell each time step.

$$
U_{i}=\sigma U_{i}^{\text {old }}+(1-\sigma) U_{i}^{\text {new }},
$$

where the superscript old denotes the value of last time step, $U_{i}^{\text {new }}$ is the arithmetic mean of information velocities of all the simulated particles within the cell during this time step, and $\sigma$ is a relaxation factor ranging between 0 and 1 .

$$
\rho=\rho_{\text {old }}+\Delta \rho,
$$

where $\Delta \rho$ is the density variation during this time step, and is obtained from the mass conservation equation

$$
\frac{\partial \rho}{\partial t}+\frac{\partial\left(\rho U_{i}\right)}{\partial x_{i}}=0 .
$$

An additional energy transfer model has been developed to update temperature in the IP method [25]. For test cases such as the thermal Couette flows over the entire flow regime, the simulated results given by IP with this model and DSMC agreed well with each other. For low subsonic flows without external heating such as those through micro-channels concerned here, the isothermal assumption is valid that allows a further simplification, i.e. temperature keeps a constant over the whole flow field. The pressure field, required in calculating the velocity increment through Eq. (2), may be obtained from the density field through the ideal gas equation of state.

In micro-channel experiments [1,5-7], the flows driven by the pressure difference between the inlet and outlet started from an initially stationary state, and gradually reached a steady state with a constant mass flux at every cross section. IP simulation undergoes the same process [20, 21]: initially assumed velocity field is corrected time step by step in order to be consistent with the local pressure gradient caused by the inlet and outlet pressure difference. The ratios of the micro-channel length to height in experiments are as large as several thousands [1,5-7], which make accumulation of numerical error inherent in a non-conservative scheme become significant in the stream-wise direction. Test calculation shows that the mass fluxes at different cross sections can not adjust and converge to a uniform steady value when the non-conservative scheme is utilized to solve Eq. (5). Thus, it is necessary to employ a conservative scheme, or, equivalently, the integral form of the mass conservation. At two-dimensional situation, a second-order center difference conservative scheme of Eq. (5) yields

$$
\Delta \rho=\Delta t\left(\frac{\rho_{i-1, j} U_{i-1, j}-\rho_{i+1, j} U_{i+1, j}}{2 \Delta x}+\frac{\rho_{i, j-1} U_{i, j-1}-\rho_{i, j+1} U_{i, j+1}}{2 \Delta y}\right) .
$$

Particle-based schemes such as DSMC and IP require a time step smaller than the mean collision time. This makes the flow field evolve quite slowly, e.g. the ratio of $\Delta \rho$ to $\rho$ is at order of $10^{-9}$. To speed up the convergent process of IP calculations, a super-relaxation technique is used

$$
\rho_{i, j}^{t+\Delta T}=\rho_{i, j}^{t}+\omega \Delta \rho_{i, j}^{t} .
$$


where $\omega$ is the super-relaxation factor. A large value is employed initially, and it gradually decreases and becomes unity to satisfy the mass conservation exactly as the stream-wise mass fluxes at different cross sections approach a steady and uniform state. The velocity of a cell given by Eq. (3) contains statistical noise that, though very small, may affect the accuracy of $\Delta \rho$ through Eq. (5). A least square technique is employed here to fit the mass fluxes of $\rho u$ and $\rho v$, respectively, each time step. This provides the smooth mass flux distributions in both $\mathrm{x}$ and $\mathrm{y}$ directions, capable of calculating $\Delta \rho$ more accurately.

\section{COMPUTATIONAL CONDITIONS}

In micro-channel experiments performed by Pong et al [1], Shih et al [5], Arkilic et al [6] and Arkilic [7], respectively, the channel width is much larger than the height (Table 1). This makes the span-wise influence negligible, and the flows can be simulated as two-dimensional. The degree of rarefaction may be measured by the Knudsen number based on the channel height. The Knudsen number at the outlet, $K n_{o}$, indicated that the experimental conditions [1,5-7] were in the slip and transition regimes, respectively.

Table 1. Flow conditions and computational parameters.*

\begin{tabular}{cccc}
\hline Case & Pong et al (1994) & Shih et al (1996) & Arkilic et al (1997) \\
\hline Gas & $\mathrm{N}_{2}$ & $\mathrm{He}$ & $\mathrm{Ar}$ \\
Height $(\mu \mathrm{m})$ & 1.2 & 1.2 & 52.3 \\
Width $(\mu \mathrm{m})$ & 40 & 40 & 7490 \\
Length $(\mu \mathrm{m})$ & 3000 & 4000 & $1.01 \times 10^{5}$ \\
$p_{o}(\mathrm{~Pa})$ & $1.01 \times 10^{5}$ & $1.01 \times 10^{5}$ & 0.05 \\
$\mathrm{Kn}$ & 0.05 & 0.135 & 0.8 \\
$\sigma_{t}$ & 1.0 & 1.0 & $700 \times 30$ \\
Cells & $300 \times 15$ & $400 \times 15$ & $5 \times 2$ \\
Subcells & $5 \times 2$ & $5 \times 2$ & \\
\hline
\end{tabular}

*Gas and channel surfaces are assumed to be consistent with all these experiments that were carried out at room temperature 294 $\mathrm{K}$.

An orthogonal coordinate system is employed (Fig. 1), with the origin at point $\mathrm{O}$, and $\mathrm{x}$ and y axes along $\mathrm{OO}^{\prime}$ and OA, respectively. Since the flows are symmetric about $\mathrm{OO}^{\prime}$, we consider a computational domain of $\mathrm{OO}^{\prime} \mathrm{BA}$ that is divided into uniform rectangular cells. Each of the cells is sub-divided into a set of uniform rectangular sub-cells within which collision pairs are selected. The numbers of cells and sub-cells are given in Table 1. The cell size is much smaller in the normal direction than in the stream-wise direction, so is the sub-cell size. As shown by Nance et al [24], the flow field is insensitive to the stream-wise cell size because of a relatively small velocity gradient in this direction. Our test calculations also verify this observation that the smaller stream-wise cell and sub-cell sizes provide the same results as the present ones. For all the cases studied in this article, the time step is equal to one third of the mean molecular collision time at the inlet, and molecular interaction is described by the variable hard-sphere (VHS) model [21]. The VHS model assumes that the scattering from molecular collision is isotropic in the center of mass frame of reference, whereas the collision diameter depends on the relative velocity. The reference collision diameter in VHS appropriate to the IP method has been determined for common gases [12]. A specular reflection is used along the symmetrical boundary $\mathrm{OO}^{\prime}$. The channel surfaces are assumed to be diffusely reflecting with a tangential momentum accommodation coefficient

$$
\sigma_{t}=\frac{u_{r}-u_{i}}{u_{s}-u_{i}}
$$




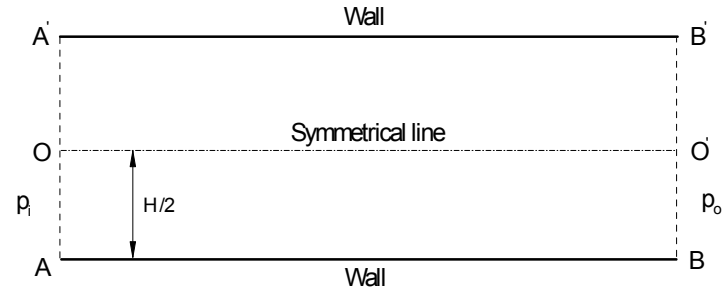

Figure 1. Computational domain of micro-channel gas flows.

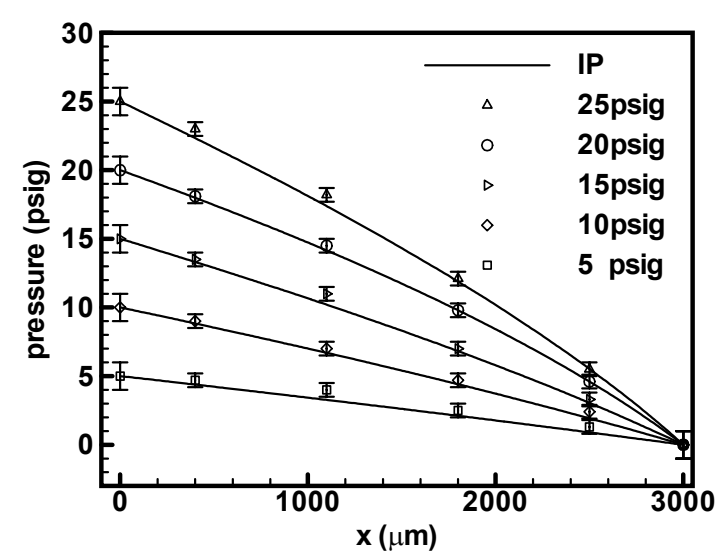

Figure 2. Comparison of stream-wise pressure distributions given by IP with data measured by Pong et al (1994).

where $u_{i}$ is the mean stream-wise velocity of incident molecules, $u_{r}$ is the mean reflected stream-wise velocity, and $u_{S}$ is the surface stream-wise velocity. The value of $\sigma_{t}$, ranging between 0 and 1 , depends upon the surface roughness and gas properties,

Arkilic et al [6,7] developed a modified accumulation technique to measure low mass flux through micro-channels. Comparing the measured mass flow rate with that of the slip Navier-Stokes solution, they extracted $\sigma_{t}$ of the micro-channel surfaces of single-crystal silicon in their system. The values appeared to range between 0.8 and 0.9, e.g. $0.80 \pm 0.01$ for argon and $0.88 \pm 0.01$ for nitrogen.

The same means was also utilized by Shih et al [5] to extract $\sigma_{t}$ for their micro-channel surfaces, which provided 0.9905 for nitrogen and 1.1620 for helium. However, the latter was beyond the physically realistic range of $\sigma_{t}$. It is known that the slip Navier-Stokes solution is no longer accurate beyond the slip regime as $K n>0.1$. In the helium experiment [5], the Knudsen number increased from 0.1 at the inlet to 0.16 at the outlet, where the extraction of $\sigma_{t}$ through the slip Navier-Stokes solution became improper. In contrast, the Knudsen number in the nitrogen experiment [5] ranged between 0.025 and 0.05 that indicated the flow was in the slip regime, and the thus obtained value of $\sigma_{t}, 0.9905$, is also reasonable. This demonstrated that the micro-channel surfaces in the UCLA system was close to the fully diffusive reflection.

\section{RESULT AND DISCUSSION}

In the first generation of experimental system for micro-channel gas flows developed by Pong et al [1], four micro pressure sensors were fabricated directly into the channel surfaces. Five inlet pressures of 5, 10, 15, 20 and 25 psig were employed, respectively, while the outlet pressure maintained at 1 atm. Figure 2 compares the stream-wise pressure distributions given by the IP method with experimental data of Pong et al [1], with the error bars to show the measured confidence limits. The simulated and experimental results agree well with each other. Because of a height of only $1.2 \mu \mathrm{m}$, the velocity gradient in the normal direction is quite large that leads to a strong viscous effect and a rapid loss of pressure in the stream-wise direction. The rarefied gas effect is clearly demonstrated by the non- 


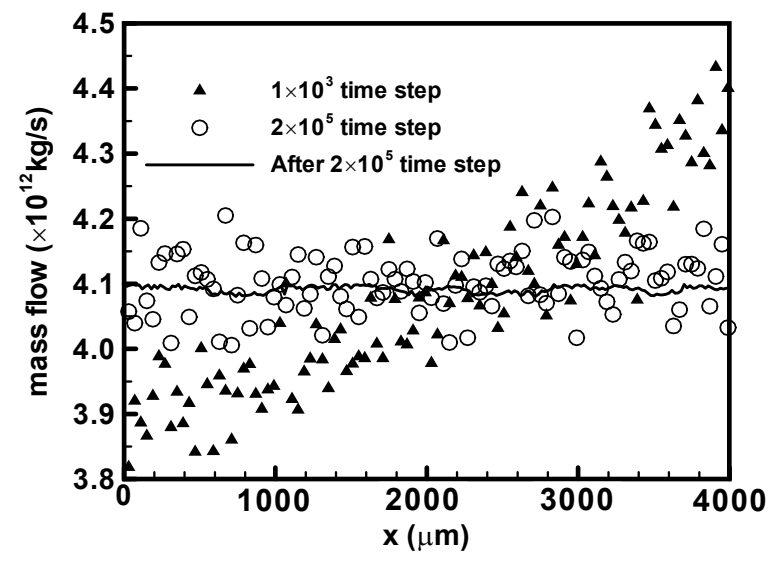

Figure 3. Evolution of mass flux distribution in IP simulation of a

flow at experimental conditions of Shih et al (1996).

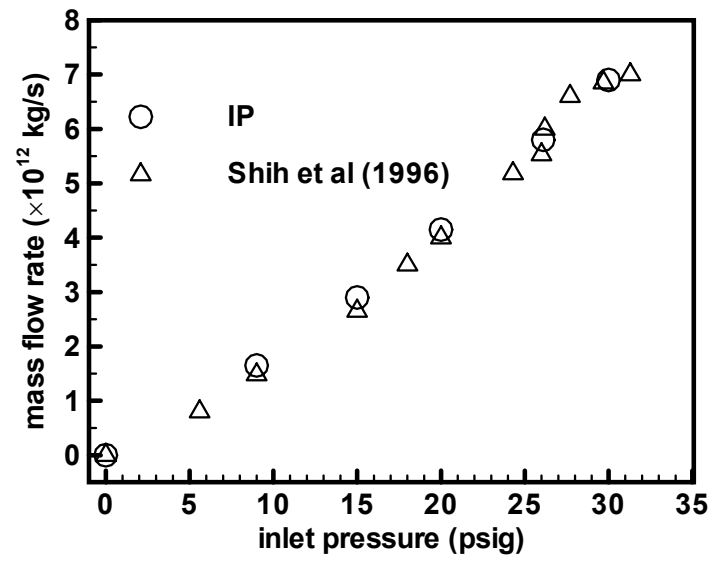

Figure 4. Relation of mass flux versus the inlet pressure in the slip and transition regimes.

linearity of the pressure profiles that become significant as the inlet pressure increases. The pressure loss is subject to the local shear stress of the micro-channel surfaces that becomes sensitive to the Knudsen number as $K n>0.01$ [12]. For the same outlet pressure, the increase of the inlet pressure results in a more significant stream-wise variation of $\mathrm{Kn}$. and therefore corresponds to a more obvious non-linear pressure profile.

In the second generation of experimental micro-channel system [5], thirteen micro pressure sensors were uniformly distributed along the channel surfaces of 4000 microns long. Figure 3 shows the evolution of mass flux of helium in IP calculating a case at an inlet pressure of $19.0 \mathrm{psig}$. The initial pressure distribution employs the slip Navier-Stokes solution. Since the flow is in the transition regime, this distribution is different from the real situation. This results in a non-uniform distribution of mass flux during the initial stage of simulation ( $1 \times 10^{3}$ time step). Using the conservative scheme (6) and the super relaxation technique (7), a steady state is approached after about $2 \times 10^{5}$ time steps, while the least-square fitting technique effectively reduces the noise and gives a relatively smooth mass-flux distribution. Then the program starts to sample. The statistical scatter becomes quite small when the sample size reaches $5 \times 10^{4}$, and the mass fluxes at different cross sections unanimously approaches $4.1 \times 10^{-12} \mathrm{~kg} / \mathrm{s}$. In Fig.4, this simulated mass flux, together with those at the inlet pressures of 8.7, 13.6, 26, and $30 \mathrm{psig}$, respectively, is compared against measured data of Shih et al [6]. There is a remarkable agreement. Figure 5 shows the stream-wise pressure distributions at three different inlet pressures given by IP and experiment, which also agree with each other.

Because of a small height at order of micron, mass flux through micro-channels is as low as $10^{-12} \mathrm{~kg} / \mathrm{s}$. Arkilic et al [6,7] developed a modified accumulation technique to measure such a low mass flux accurately. Figure 6 compares the mass flow rates of nitrogen at an outlet Knudsen number of 0.05 given by IP calculations and Arkilic et al experiment [6]. The flows were in the slip regime, and a remarkable agreement can be seen.

In addition to various experimental conditions, a two-dimensional short micro-channel is investigated over the entire Knudsen regime from continuum to free molecular using the IP and DSMC methods. The micro-channel is $1 \mu m$ high and $15 \mu m$ long. The normalized mass flow rates as functions of the Knudsen number at the inlet $K n_{i}$ are shown in Fig. 7, where the ratio of the outlet to inlet pressure is 0.7 , the normalized factor of mass flux is $\left(\rho_{i}+\rho_{o}\right) v_{m} H / 2, \rho_{i}$ and $\rho_{o}$ are the inlet and outlet densities, respectively, and $v_{m}=\sqrt{2 R T}$ is the most probable 


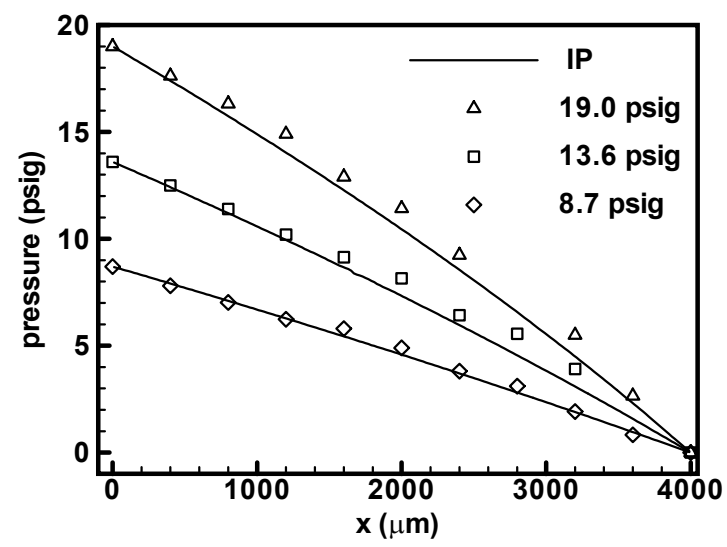

Figure 5. Comparison of stream-wise pressure distributions given by IP with data measured by Shih et al (1996).

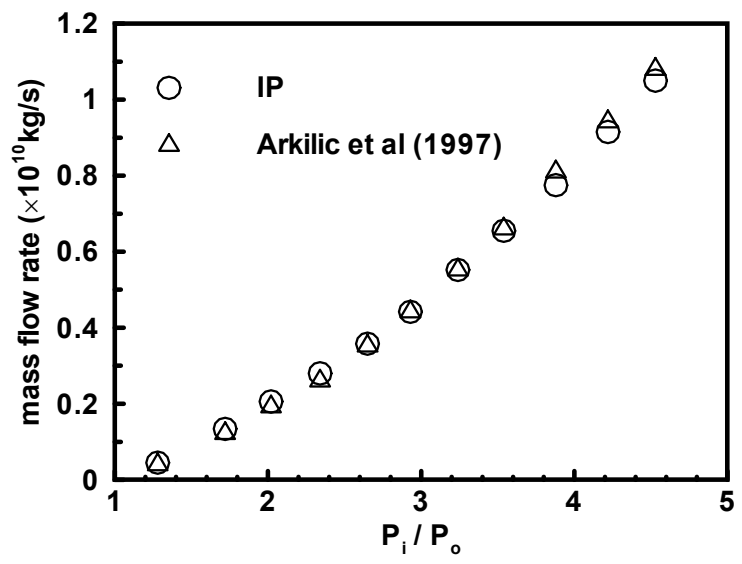

Figure 6. Variation of mass flux versus the ratio of the inlet to outlet pressure in the slip regime.

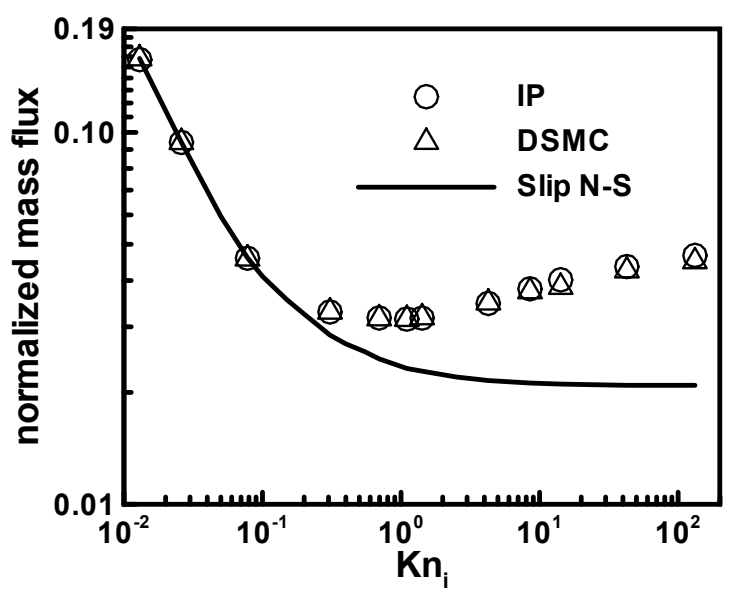

Figure 7. Relation of the normalized mass flux versus the inlet Knudsen number $\left(K n_{i}\right)$ for a short micro-channel.

thermal speed. The IP and DSMC results are in excellent agreement over the entire Knudsen regime, and demonstrate a minimum around $K n_{i}$ of 1 . This phenomenon was first observed by Knudsen [26], and therefore is referred to as the Knudsen minimum. The slip Navier-Stokes solution deviates from IP and DSMC results in the transition regime and fails to predict the Knudsen minimum.

\section{ACKNOWLEDGMENTS}

This work was supported by Chinese Academy of Sciences under grant KJ 951-1-202, and National Natural Science Foundation of China under grants 19382009,19889209.

\section{RFERENCES}

1. Pong, K. C., Ho, C. M., Liu, J. Q., and Tai, Y. C., Non-linear pressure distribution in uniform micro-channels, ASME-FED, 197, 51 (1994). 
2. Arkilic, E. B., Breuer, K., and Schmidt, M. A., Gaseous slip flow in long micro-channels, J. MicroElectroMechanical Systems 6, 167 (1997).

3. Harley, J. C., Huang, Y., Bau, H., and Zemel, J. N., Gas flow in micro-channels, J. Fluid Mech., 248, 257(1995).

4. Shih, J. C., Ho, C. M., Liu, J., and Tai, Y. C., Non-linear pressure distribution in uniform microchannels, ASME-AMD 238 (1995).

5. Shih, J. C., Ho, C. M., Liu, J. Q., and Tai, Y. C., Monatomic and polyatomic gas flow through uniform microchannels, ASME-DSC 59, 197 (1996).

6. Arkilic, E. B., Schmidt, M. A., and Breuer, K. S., Measurement of the TMAC in silicon microchannels, in Rarefied Gas Dynamics, edited by C. Shen, Peking University Press, 983 (1997).

7. Arkilic, E. B., Measurement of the mass flow and tangential momentum accommodation coefficient in silicon microchannels, Ph.D. thesis, MIT, FDRL TR 97-1(1997).

8. Ho, C. M. and Tai, C. Y., Micro-electro-mechanical-systems (MEMS) and fluid flows, Annu. Rev. Fluid Mech., 30, 579(1998).

9. Beskok, A. and Karniadakis, G., Rarefaction and compressibility effects in gas microflows, J. Fluids Engin., 11, 448(1996).

10. Oran, E. S., Oh, C. K., and Cybyk, B. Z., Direct simulation Monte Carlo: recent advances and applications, Annu. Rev. Fluid Mech., 30, 403 (1998).

11. Fan, J. and Shen, C., Statistical simulation of low-speed unidirectional flows in transitional regime, In Rarefied Gas Dynamics, edited by R Brun, R Campargue, R Gatignol, J C Lengrand, Cepadues Editions, 2, 245 (1999).

12. Fan, J. and Shen, C., Statistical simulation of low-speed rarefied gas flows, J. Computational Physics, 167, 393 (2001).

13. Dong, W., University of California Report UCRL-3353, (1956).

14. Ohwada, T., Sone, Y., and Aoki, K., Numerical analysis of the Poiseuille flow and thermal transpiration flows between two parallel plates on the basis of the linearized Boltzmann equation for hard-sphere molecules, Phys. Fluids, 1, 2042 (1989).

15. Sone, Y., Takata, S., and Ohwada, T., Numerical analysis of the plane Couette flow of a rarefied gas on the basis of the linearized Boltzmann equation for hard-sphere molecules, European J. Mech., B/Fluids, 9, 273 (1990).

16. Sun, Q., Boyd, I. D., and Cadnler, G. V., Numerical simulation of gas flow over micro-scale airfoils, AIAA 2001-3071 (2001).

17. Schaff, S. A. and Sherman, F. S., Skinfriction in slip flow, J. Aero Sci. 21, 85 (1954).

18. Janour, Z., Resistances of a plate in parallel flow at low Reynolds numbers, NACA TM 1316 (1954).

19. Cai, C. P., Boyd, I. D., Fan, J., and Candler, G., Direction simulation methods for low-speed microchannel flows, $J$. Thermophysics and Heat Transfer, 14, 368 (2000).

20. Xie, C., Fan, J., and Shen, C., Statistical simulation of micro-channel gas flows. in Proceedings of Mass and Heat Transfer of Chinese Society of Engineering Thermophysics, 388 (2000).

21. Bird, G.A., Molecular Gas Dynamics and the Direct Simulation of Gas Flow, Clarendon Press (1994).

22. Fan, J., Boyd, I.D., Cai, C.P., Hennighausen, K., and Candler, G.V., Computation of rarefied gas flows around a NACA 0012 airfoil. AIAA J., 2001, 39, 618 (2001).

23. Shen, C., Jiang, J. Z., and Fan, J., Information preservation method for the case of temperature variation, in Rarefied Gas Dynamics, edited by T.J. Bartel and M.A. Gallis, AIP, 185 (2001).

24. Nance, R. P., Hash, D., and Hassan, H. A., Role of boundary conditions in Monte Carlo simulation of MEMS devices, $J$. Thermophysics and Heat Transfer, 11, 497 (1997).

25. Sun, Q.H., Boyd, I.D., A direct simulation method for subsonic, microscale gas flows, J. Comp. Phys., 179, 400 (2002).

26. M. Knudsen. Ann. Physic, 28, 75 (1909). 